\title{
Comparison of the protective effects of steamed and cooked broccolis on ischaemia-reperfusion-induced cardiac injury
}

\author{
Subhendu Mukherjee ${ }^{1,2}$, Istvan Lekli ${ }^{1}$, Diptarka Ray ${ }^{1}$, Hiranmoy Gangopadhyay ${ }^{2}$, Utpal Raychaudhuri ${ }^{2}$ \\ and Dipak K. Das ${ }^{1 *}$ \\ ${ }^{1}$ Cardiovascular Research Center, University of Connecticut School of Medicine, Farmington, CT 06030-1110, USA \\ ${ }^{2}$ Center for Medicinal Food and Applied Nutrition, Jadavpur University, Kolkata, India
}

(Received 5 May 2009 - Revised 10 September 2009 - Accepted 11 September 2009 - First published online 27 October 2009)

Recently, broccoli, a vegetable of the Brassica family, has been found to protect the myocardium from ischaemic reperfusion injury through the redox signalling of sulphoraphane, which is being formed from glucosinolate present in this vegetable. Since cooked broccoli loses most of its glucosinolate, we assumed that fresh broccoli could be a superior cardioprotective agent compared to cooked broccoli. To test this, two groups of rats were fed with fresh (steamed) broccoli or cooked broccoli for $30 \mathrm{~d}$, while a third group was given vehicle only for the same period of time. After $30 \mathrm{~d}$, all the rats were sacrificed, and the isolated working hearts were subjected to 30 min ischaemia followed by $2 \mathrm{~h}$ of reperfusion. Both cooked and steamed broccolis displayed significantly improved post-ischaemic ventricular function and reduced myocardial infarction and cardiomyocyte apoptosis compared to control, but steamed broccoli showed superior cardioprotective abilities compared with the cooked broccoli. Corroborating with these results, both cooked and steamed broccolis demonstrated significantly enhanced induction of the survival signalling proteins including Bcl2, Akt, extracellular signal-regulated kinase 1/2, haemoxygenase-1, NFE2 related factor 2, superoxide dismutase (SOD1) and SOD2 and down-regulation of the proteins (e.g. Bax, c-Jun N-terminal kinase, p38 mitogen-activated protein kinase) of the death signalling pathway, steamed broccoli displaying superior results over its cooked counterpart. The expressions of proteins of the thioredoxin (Trx) superfamily including Trx 1 and its precursor sulphoraphane, Trx 2 and Trx reductase, were enhanced only in the steamed broccoli group. The results of the present study documented superior cardioprotective properties of the steamed broccoli over cooked broccoli because of the ability of fresh broccoli to perform redox signalling of Trx.

Broccoli: Cardioprotection: Ischaemia/reperfusion: Sulphoraphane

Broccoli, a vegetable of cabbage family of genus Brassica, has been used as a vegetable from time immemorial. It is rich in vitamin $\mathrm{C}$, vitamin $\mathrm{K}$, vitamin $\mathrm{A}$ as well as dietary fibre and certain minerals, but lesser amounts of fat and energy. For these reasons, broccoli has become a vegetable of choice in modern society. Existing studies suggest that it can reduce the risk of cancer by blocking DNA damage ${ }^{(1)}$ and its juices (from leaves) can prevent skin disease ${ }^{(2)}$. Available evidence indicates that the protective effects of broccoli are indicated through the inhibition of cellular oxidative stress $^{(3)}$ and reduction of cholesterol ${ }^{(4)}$. Recent studies have indicated that broccoli sprouts can induce carcinogendetoxifying enzymes ${ }^{(5)}$ and broccoli can protect the mammalian heart through the activation of the genes of thioredoxin (Trx) superfamily ${ }^{(6,7)}$.

Epidemiological studies suggest that broccoli contains certain phytochemicals that may confer protection against some degenerative diseases like cancer ${ }^{(8,9)}$ and $\mathrm{CVD}^{(6)}$. Apart from the above-mentioned beneficial components, broccoli is a good source of dietary selenium and sulphoraphane ${ }^{(10)}$, the latter being an isothiocyanate, which possess anti-carcinogenic effects ${ }^{(11,12)}$. It is also known as a natural inducer of phase II enzymes like haemoxygenase-1 (HO-1) in both human and animals ${ }^{(13,14)}$. Sulphoraphane can induce redoxregulated cardioprotective protein Trx through the antioxidant-responsive element ${ }^{(15)}$. A study has shown that broccoli protects the mammalian heart via the activation of Trx superfamily genes ${ }^{(7)}$. Although a major part of the health beneficiary effects of broccoli is due to the presence of sulphoraphane, broccoli does not contain sulphoraphane as such, rather it contains high concentration of glucosinolate, a $\beta$-thioglucoside $N$-hydroxysulphate. Broccoli also contains myrosinase, a thioglucosidase but not in direct contact with its substrate. Upon crushing, the cellular membranes get ruptured, and its cellular constituents come to contact with glucosinolate. Glucosinolate is then hydrolysed via the enzymatic activity of myrosinase to form an unstable intermediate thiohydroamate- $O$-sulphonate, which in turn is converted to a number of metabolites like isothiocyanate, thiocyanate, nitriles etc. (Fig. 1) ${ }^{(16,17)}$. Sulphoraphane is the major isothiocyanate

Abbreviations: ERK, extracellular signal-regulated kinase; HO-1, haemoxygenase-1; JNK, c-Jun N-terminal kinase; LVDP, left ventricular developed pressure;

MAPK, mitogen-activated protein kinase; Nrf2, NFE2 related factor 2; SOD, superoxide dismutase; Trx, thioredoxin.

* Corresponding author: Dipak K. Das, fax +1 860679 4606, email ddas@neuron.uchc.edu 


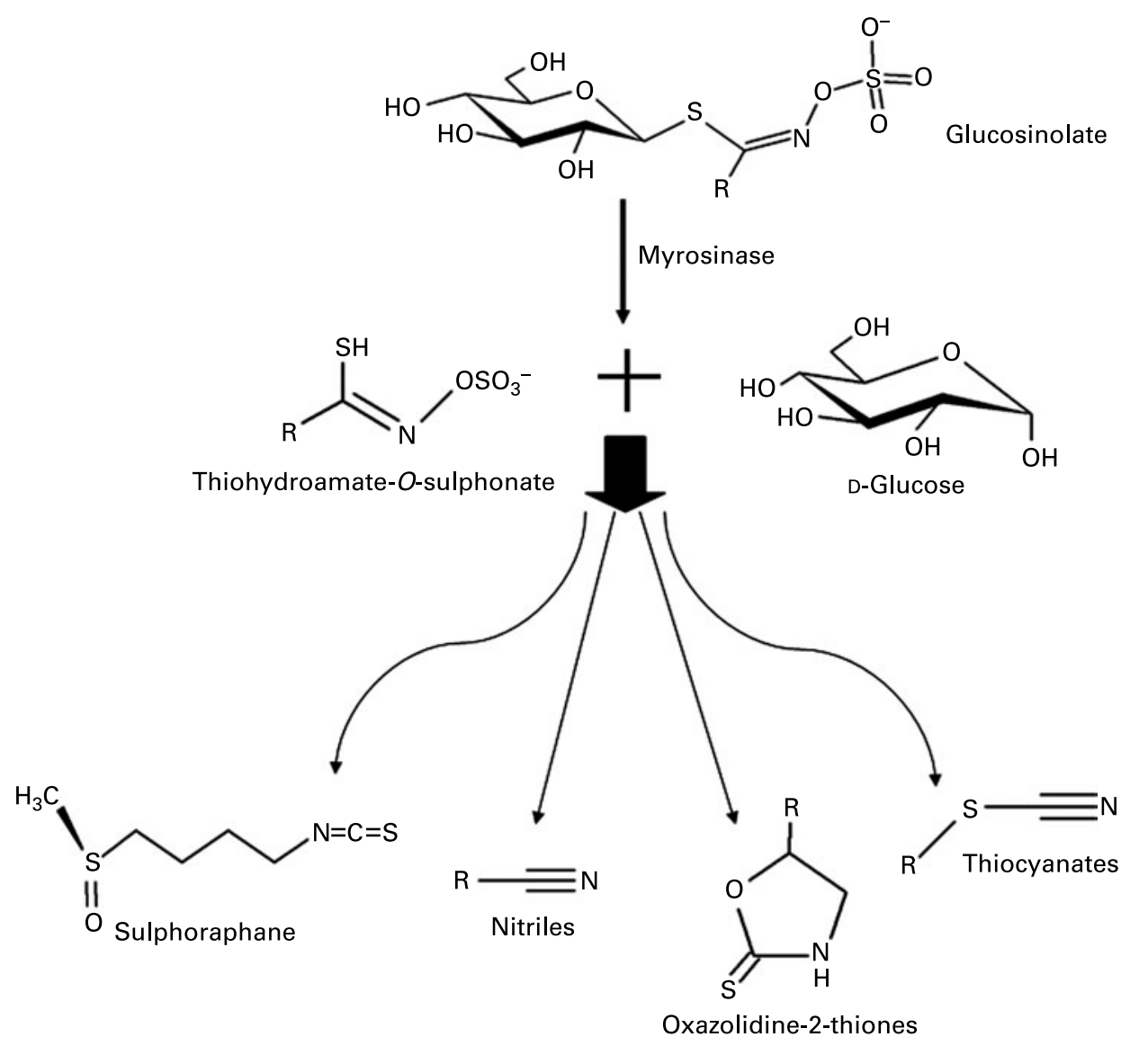

Fig. 1. Chemical reactions of formation of isothiocyanate from broccoli.

present in broccoli. Studies have shown that enzymatic activity of myrosinase decreases with temperature elevation ${ }^{(18)}$; and its activity remains stable up to $40^{\circ} \mathrm{C}$, but is reduced by $90 \%$ when heated for $10 \mathrm{~min}$ at $60^{\circ} \mathrm{C}^{(19)}$. The stability of sulphoraphane is also temperature sensitive. A study demonstrated that sulphoraphane is degraded more than $90 \%$ after $20 \mathrm{~min}$ of heating at $90^{\circ} \mathrm{C}^{(19)}$. On the other hand, some studies have shown that cooking has no effect on the antioxidant properties of broccoli ${ }^{(20)}$. Based on these results, we hypothesised that fresh broccoli might possess additional health benefits compared to cooked broccoli. To test this hypothesis, we fed a group of rats steamed broccoli, while another group was given the cooked broccoli. After $30 \mathrm{~d}$, isolated hearts from the experimental and control rats were subjected to $30 \mathrm{~min}$ ischaemia followed by $2 \mathrm{~h}$ of reperfusion. Cardiac functional parameters like heart rate, left ventricular developed pressure (LVDP), LVdp/dt (first derivative of LVDP), coronary flow and aortic flow were measured. Also some proteins in the death/survival signalling pathways (Bax, Bcl2, Bcl-xL Akt, extracellular signal-regulated kinase (ERK) 1/2, c-Jun N-terminal kinase (JNK), NFE2 related factor 2 (Nrf2), HO-1), superoxide dismutase (SOD1), SOD2 etc.) were measured. The expression level of some Trx superfamily proteins such as Trx, Trx 2 and Trx reductase (TrxR) was measured. The results of the present study clearly demonstrated superior cardioprotective effects of steamed broccoli compared to its cooked counterpart.

\section{Materials and methods}

Preparation of broccoli slurry

Broccoli was purchased from local supermarket. Fresh broccoli was blanched by subjecting it to steaming, and then a slurry was made with adding water in a ratio of $1: 3.5(\mathrm{w} / \mathrm{w})$ and mixing in a blender. Cooked broccoli preparation was done in a similar way by mixing in a blender and slurry was prepared with water (in the same ratio), and then the slurry was heated in microwave at high temperature setting for total $20 \mathrm{~min}$.

\section{Animals}

All animals used in the present study received humane care in compliance with the Animal Welfare Act and other federal statutes and regulations relating to animals and experiments involving animals and adheres to principles stated in the Guide for the Care and Use of Laboratory Animals, NRC Publication, 1996 edition. Sprague-Dawley male rats weighing between 250 and $300 \mathrm{~g}$ were used for the experiment. The rats were randomly assigned to one of the three groups: (1) control (2), steamed broccoli and (3) cooked broccoli (Fig. 2). The rats were fed ad libitum regular rat chow with free access to water. The broccoli (both steamed broccoli and cooked broccoli)-treated rats were fed with $1 \mathrm{ml}$ broccoli slurry $(1.5 \mathrm{~g} / \mathrm{kg}$ body weight per $\mathrm{d})$ for $30 \mathrm{~d}$, while the control group of rats were gavaged $1 \mathrm{ml}$ water for the same period of time. 


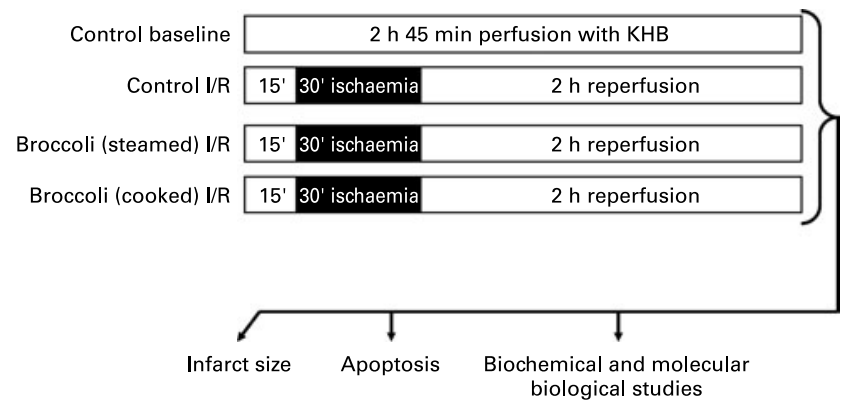

Fig. 2. Schematic representation of experimental perfusion protocol of different groups of heart. KHB, Krebs-Henseleit bicarbonate buffer; I/R, ischaemia/reperfusion.

\section{Isolated working heart preparation}

At the end of $30 \mathrm{~d}$, the rats were anaesthetised with sodium pentobarbital $(80 \mathrm{mg} / \mathrm{kg}$ body weight, intraperitoneal; Abbott Laboratories, North Chicago, IL, USA) and anticoagulated with heparin sodium $(5 \mathrm{mg}(500 \mathrm{IU}) / \mathrm{kg}$ body weight, intravenous; Elkins-Sinn Inc., Cherry Hill, NJ, USA) injection. After ensuring sufficient depth of anaesthesia, thoracotomy was performed; the hearts were perfused in the retrograde Langendorff mode at $37^{\circ} \mathrm{C}$ at a constant perfusion pressure of $100 \mathrm{~cm}$ of water $(10 \mathrm{kPa})$ for a $5 \mathrm{~min}$ washout period ${ }^{(7)}$. The perfusion buffer used in the present study consisted of a modified Krebs-Henseleit bicarbonate buffer (in mM: $\mathrm{NaCl} 118, \mathrm{KCl}$ $4 \cdot 7$, calcium chloride $1 \cdot 7$, sodium bicarbonate 25 , potassium biphosphate $0 \cdot 36$, magnesium sulphate 1.2 and glucose 10 ). The Langendorff preparation was switched to the working mode following the washout period. At the end of $10 \mathrm{~min}$, after the attainment of steady-state cardiac function, baseline functional parameters were recorded. Then the hearts were subjected to $30 \mathrm{~min}$ of global ischaemia followed by $2 \mathrm{~h}$ of reperfusion. The first $10 \mathrm{~min}$ of reperfusion was in the retrograde mode to allow for post-ischaemic stabilisation and thereafter, in the antegrade working mode to allow for assessment of functional parameters, which were recorded at 30,60 and $120 \mathrm{~min}$ of reperfusion.

\section{Cardiac function assessment}

Aortic pressure was measured using a Gould P23XL pressure transducer (Gould Instrument Systems Inc., Valley View, $\mathrm{OH}$, USA) connected to a side arm of the aortic cannula, and the signal was amplified using a Gould 6600 series signal conditioner and monitored on a CORDAT II real-time data acquisition and analysis system (Triton Technologies, San Diego, CA, USA) ${ }^{(7)}$. Heart rate, LVDP (defined as the difference of the maximum systolic and diastolic aortic pressures) and the first derivative of developed pressure $(\mathrm{dp} / \mathrm{dt})$ were all derived or calculated from the continuously obtained pressure signal. aortic flow was measured using a calibrated flow meter (Gilmont Instrument Inc., Barrington, IL, USA), and coronary flow was measured by timed collection of the coronary effluent dripping from the heart.

\section{Infarct size estimation}

At the end of reperfusion, a $1 \%(\mathrm{w} / \mathrm{v})$ solution of triphenyl tetrazolium chloride in phosphate buffer was infused into aortic cannula for $20 \mathrm{~min}$ at $37^{\circ} \mathrm{C}^{(21)}$. The hearts were excised, and the sections $(0.8 \mathrm{~mm})$ of the heart were fixed in $2 \%$ paraformaldehyde, placed between two cover slips and digitally imaged using a Microtek Scan Maker 600z. To quantify the areas of interest in pixels, a NIH image 5.1 (a public-domain software package) was used. The infarct size was quantified and expressed in pixels.

\section{Terminal deoxynucleotidyl transferase dUTP nick end labelling assay for assessment of apoptotic cell death}

Immunohistochemical detection of apoptotic cells was carried out using the terminal deoxynucleotidyl transferase dUTP nick end labelling method ${ }^{(21)}$ (Promega, Madison, WI, USA). The heart tissues were immediately put in $10 \%$ formalin and fixed in an automatic tissue-fixing machine. The tissues were carefully embedded in the molten paraffin in metallic blocks, covered with flexible plastic moulds and kept under freezing plates to allow the paraffin to solidify. The metallic containers were removed and tissues became embedded in paraffin on the plastic moulds. Before analysing tissues for apoptosis, tissue sections were deparaffinised with xylene and washed in succession with different concentrations of ethanol (absolute, 95, 85, 70 and 50\%). Then the transferase-mediated dUTP nickend labeling staining was performed according to the manufacturer's instructions. The fluorescence staining was viewed with a fluorescence microscope (AXIOPLAN2 IMAGING; Carl Zeiss Microimaging Inc., Thornwood, NY, USA) at $520 \mathrm{~nm}$ for green fluorescence of fluorescein and at $620 \mathrm{~nm}$ for red fluorescence of propidium iodide. The number of apoptotic cells was counted throughout the slides and expressed as a percent of total myocyte population.

\section{Preparation of subcellular fractions}

Tissues (frozen in liquid nitrogen and stored at $-80^{\circ} \mathrm{C}$ ) were homogenised in $1 \mathrm{ml}$ buffer A $(25 \mathrm{~mm}$ Tris $-\mathrm{HCl}, \mathrm{pH} 8$, $25 \mathrm{~mm} \mathrm{NaCl}, 1 \mathrm{~mm}$ sodium-orthovanadate, $10 \mathrm{~mm} \mathrm{NaF}$, $10 \mathrm{~mm}$ sodium-pyrophosphate, $10 \mathrm{~nm}$ okadaic acid, $0.5 \mathrm{~mm}$ EDTA, $1 \mathrm{~mm}$ phenylmethylsulphonyl fluoride and $1 \times$ protease inhibitor cocktail) in a Polytron homogeniser. Homogenates were centrifuged at $2000 \mathrm{rpm}$ at $4^{\circ} \mathrm{C}$ for $10 \mathrm{~min}$, and the nuclear pellet was resuspended in $500 \mu \mathrm{l}$ buffer $\mathrm{A}$ with $0.1 \%$ Triton X-100 and $500 \mathrm{~mm} \mathrm{NaCl}$. Supernatant from the above centrifugation was further centrifuged at $10000 \mathrm{rpm}$ at $4^{\circ} \mathrm{C}$ for $20 \mathrm{~min}$, and the resultant supernatant was used as cytosolic extract. The mitochondrial pellet from the above centrifugation was resuspended in 200-300 $\mu \mathrm{l}$ buffer A with $0 \cdot 1 \%$ Triton X-100. The nuclear and mitochondrial pellets were lysed by incubation for $1 \mathrm{~h}$ on ice with intermittent tapping. Homogenates were then centrifuged at $14000 \mathrm{rpm}$ at $4^{\circ} \mathrm{C}$ for $10 \mathrm{~min}$, and the supernatant was used as nuclear and mitochondrial lysate, respectively. Cytosolic, nuclear and mitochondrial extracts were aliquoted, snap frozen and stored at $-80^{\circ} \mathrm{C}$ for subsequent use. Total protein concentrations in cytosolic, nuclear and mitochondrial extracts were determined using a BCA Protein Assay Kit (Pierce, Rockford, IL, USA). 


\section{Western blot analysis}

The proteins from cytosolic, nuclear or mitochondrial fractions were separated in SDS-PAGE and transferred to nitrocellulose filters. Filters were blocked in $5 \%$ non-fat dry milk, and probed with primary antibody for overnight $^{(21)}$. Primary antibodies such as Trx, Trx2, TrxR, Nrf2, SOD2 and glyceraldehyde-6-phosphate dehydrogenase were obtained from Santa Cruz Biotechnology (Santa Cruz, CA, USA), whereas Bax, Bcl2, Bcl-xL, cytochrome c, HO-1, Akt, phospho-Akt, SOD1, p38, phospho-p38, ERK, phospho-ERK, JNK, phospho-JNK, histone and Cox4 were obtained from Cell Signaling Technology (Beverly, MA, USA). All primary antibodies were used at the dilution of 1:1000. Protein bands were identified with horseradish peroxidase-conjugated secondary antibody (1:2000 dilution) and Western blotting Luminol Reagent (Santa Cruz Biotechnology). Glyceraldehyde-6-phosphate dehydrogenase, histone and Cox4 were used as cytosolic, nuclear and mitochondrial loading controls, respectively. The resulting blots were digitised, subjected to densitometric scanning using a standard NIH image program and normalised against loading control.

\section{Statistical analysis}

The values for myocardial functional parameters, total and infarct volumes and infarct sizes as well as cardiomyocyte apoptosis are all expressed as the means with their standard errors. ANOVA test followed by Bonferroni's correction was first carried out to test for any differences between the mean values of all groups. If differences between groups were established, the values of the treated groups were compared to those of the control group by a modified $t$ test. The results were considered significant if $P<0 \cdot 05$.

\section{Results}

Comparison of the effects of steamed broccoli and cooked broccoli on left ventricular function

We compared the efficacy of steamed broccoli $v$. cooked broccoli as a cardioprotectant. Although both the steamed broccoli and cooked broccoli showed superior ventricular performance compared to control rats during the reperfusion phase (Fig. 3), the steamed broccoli displayed significantly greater recovery of aortic flow, LVDP and the maximum
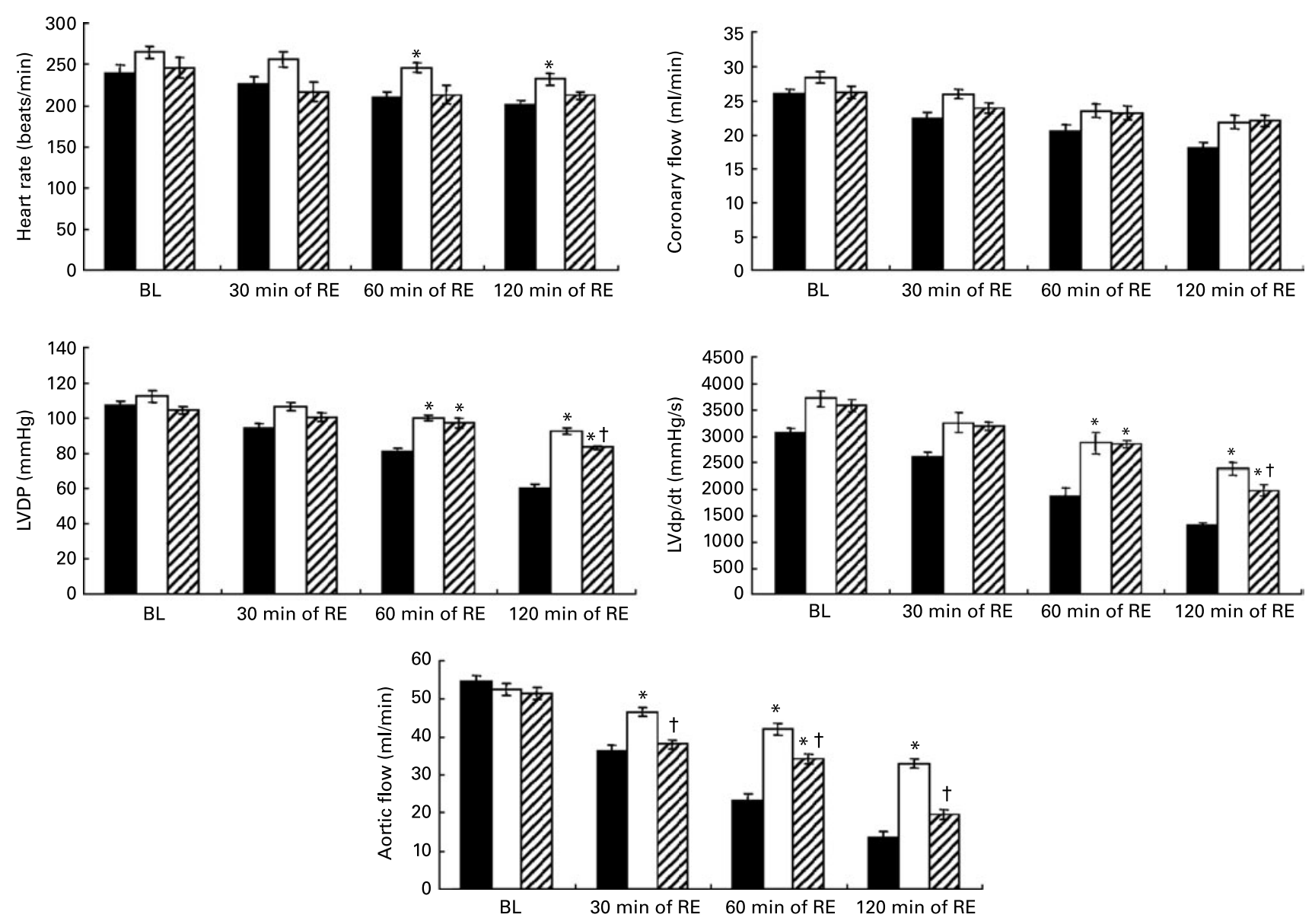

Fig. 3. Effect of steamed and cooked broccolis on post-ischaemic left ventricular function, heart rate, coronary flow, left ventricular developed pressure (LVDP), maximum first derivative of developed pressure and aortic flow. Results are shown as means with their standard errors. ${ }^{*} P<0.05 v$. ischaemic control and $\dagger P<0.05 v$. steamed broccoli. $n 6$ in each group. BL, baseline; RE, reperfusion. $\mathbf{\square}$, Control; $\square$, steamed broccoli; $\mathbb{2}$, cooked broccoli. 
first derivative of developed pressure $\left(\mathrm{LV}_{\max } \mathrm{dp} / \mathrm{dt}\right)$ compared to that achieved from cooked broccoli. The heart rate and coronary flow did not vary between the groups.

\section{Effects of steamed broccoli and cooked broccoli on myocardial infarction}

Myocardial infarct size determined by triphenyltetrazolium chloride staining was about 40.96 (SD 1.29) \% in case of ischaemia/reperfusion control group (Fig. 4). No infarction was found when the hearts were perfused with the Krebs-Henseleit bicarbonate buffer without subjecting to ischaemia and reperfusion protocol (baseline control). Both the steamed and cooked broccoli groups showed lower infarct size compared to ischaemia/reperfusion control, but steamed broccoli displayed smaller infarct size (18.82 (SD 1.24)\%) than that of the cooked broccoli treated group (23.44 (SD 1.00$) \%$ ).

\section{Effects of steamed broccoli and cooked broccoli on cardiomyocyte apoptosis}

Cardiomyocyte apoptosis was determined by transferasemediated dUTP nickend labeling method. As shown in Fig. 5(a), it was about 36.59 (SD 2.38) \% at the end of reperfusion in case of ischaemia/reperfusion control group. There were insignificant numbers of apoptotic cells (8.4 (SD 2)\%) in the hearts perfused with the Krebs-Henseleit bicarbonate buffer without subjecting to ischaemia and reperfusion (baseline control group). Both types of broccoli (steamed and cooked) treatments demonstrated substantial reduction in the number of apoptotic cells compared to non-treated control group (17.74 (SD 1.13) \% and 22.86 (SD 2.28) \% for steamed broccoli and cooked broccoli, respectively).

In order to further confirm that steamed broccoli is better in prevention of cardiomyocyte apoptosis, we determined the activity of cytochrome c. Fig. 5(b) shows the results.

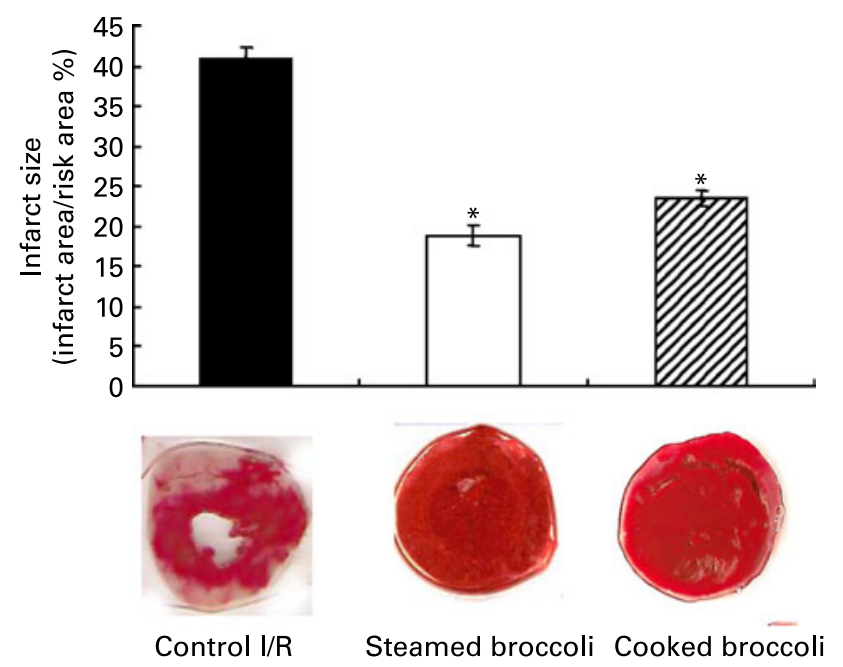

Fig. 4. Effect of steamed and cooked broccolis on myocardial infarct size. Results are shown as means with their standard errors. * $P<0.05 \mathrm{v}$. ischaemic control, $n 3$ in each group. $\square$, Control; $\square$, steamed broccoli; $\mathbb{Z}$, cooked broccoli. I/R, ischaemia/reperfusion.
Cytochrome c was significantly increased after ischaemia/ reperfusion in case of non-treated ischaemic control group, which correlates with the results of Fig. 5(a). Steamed broccoli prevented the increase in cytochrome $\mathrm{c}$ due to ischaemia, but cooked broccoli did not prevent the activation of cytochrome c after ischaemia.

\section{Effects of steamed and cooked broccolis in the generation of survival signal}

It is well known that ischaemia/reperfusion causes cellular injury by generating a death signal or by preventing survival signal $^{(22)}$. For example, it is known that ischaemia/reperfusion injury increases pro-apoptotic protein Bax and reduces antiapoptotic protein $\mathrm{Bcl} 2$. We, therefore, determined these proand anti-apoptotic parameters and survival signalling members in the hearts with and without broccoli treatment. As shown in Fig. 6(a), both steamed and cooked broccolis had the ability to enhance the phosphorylation of Akt severalfold, but steamed broccoli had higher potential than cooked broccoli.

Similarly, ischaemia/reperfusion increased pro-apoptotic Bax and reduced anti-apoptotic $\mathrm{Bcl} 2$ and $\mathrm{Bcl}-\mathrm{xL}$ as expected (Fig. 6(a)). While broccoli groups increased the $\mathrm{Bcl} 2$ and $\mathrm{Bcl}-\mathrm{xL}$ levels, the greater degree of enhancement in $\mathrm{Bcl} 2$ expression was noticed in steamed broccoli group. Bax protein level was also lower in steamed broccoli group. Taken together, these results indicate that although broccoli generates an overall survival signal, steamed broccoli has better efficacy than cooked broccoli in suppressing pro-apoptotic proteins and boosting the anti-apoptotic proteins.

\section{Effects of steamed and cooked broccolis on mitogen-activated protein kinase signalling}

It is known that ischaemia/reperfusion-induced death signal is produced from defective mitogen-activated protein kinase (MAPK)/tyrosine kinase signalling ${ }^{(21)}$. We, therefore, determined the effect of broccoli on MAPK signalling in the hearts. As shown in Fig. 6(b), both steamed and cooked broccolis had the ability to enhance the phosphorylation of ERK1/2 severalfold, but steamed broccoli possessed higher capacity to enhance the phosphorylation of ERK1/2 than that of the cooked broccoli. An opposite phosphorylation pattern was observed for proapoptotic JNK and p38 MAPK with broccoli treatment, wherein steamed broccoli showed superiority in reduction of death signal over cooked broccoli.

\section{Effects of steamed and cooked broccolis on the expression of thioredoxin proteins}

It is known that broccoli induced survival signal and reduced death signal via the activation of Trx superfamily genes ${ }^{(7)}$, and sulphoraphane is a well-known inducer of $\operatorname{TrxR}^{(23)}$. We checked whether cooking broccoli has any effect on the expression of some Trx superfamily protein such as Trx (whole), TrxR (Fig. 7(a)) and Trx2 (Fig. 7(b)). As shown in Fig. 7, both types of broccoli preparation can prevent the reduction of Trx (whole), Trx 2 and TrxR protein level due to ischaemia/reperfusion injury, but steamed broccoli showed superiority over cooked broccoli. 
(a)

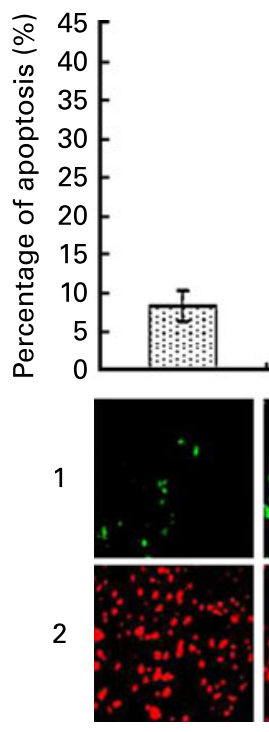

Control BL
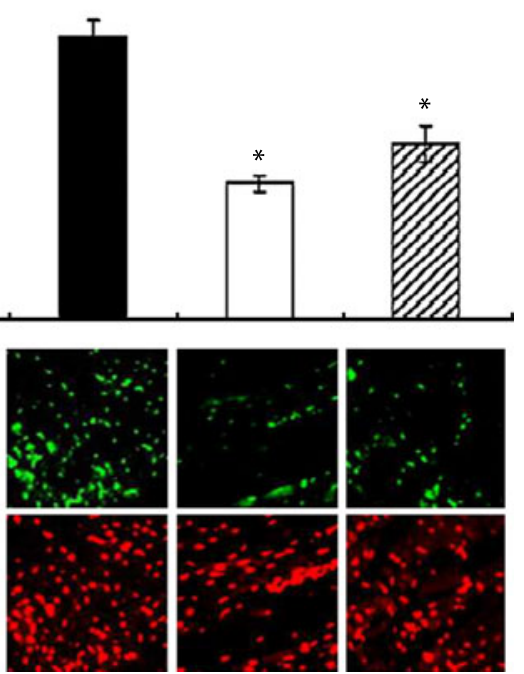

Control I/R

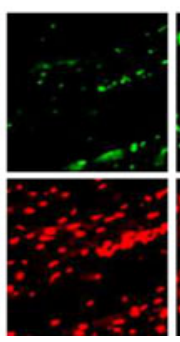

Steamed broccoli

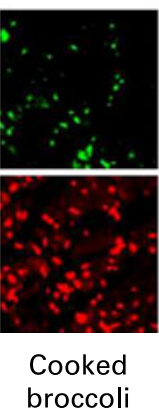

(b)

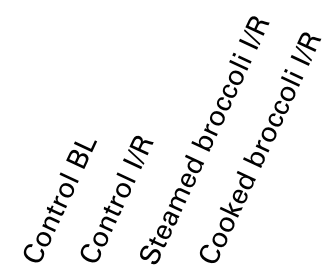

Cytochrome GAPDH

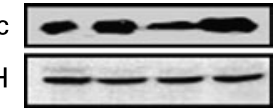

Fig. 5. Effect of steamed and cooked broccolis on cardiomyocyte apoptosis (a) and Western blot analysis of cytochrome c from the cytosolic fraction of control baseline (BL), ischaemic control, steamed and cooked broccoli-treated heart samples. Glyceraldehyde-6-phosphate dehydrogenase (GAPDH) was used as the loading control (b). In Fig. 5(a), panel 1 is total number of apoptotic cells (green channels). Panel 2 is total number of cells (red channel). Values are means with

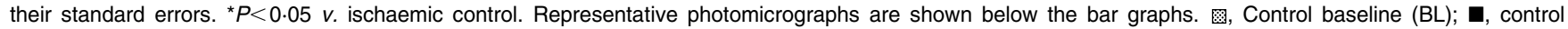
ischaemia/reperfusion (I/R); $\square$, steamed broccoli; 尚, cooked broccoli.

Effects of steamed and cooked broccolis on the expression of NFE2 related factor 2 and haemoxygenase-1 proteins

Since antioxidants present in broccoli and the ability of inducing phase II antioxidant detoxifying enzyme have been attributed to the cardioprotective effects of broccoli, we compared the effects of fresh broccoli and cooked broccoli treatment on Nrf2 and phase II detoxifying enzyme HO-1 expression, (a)

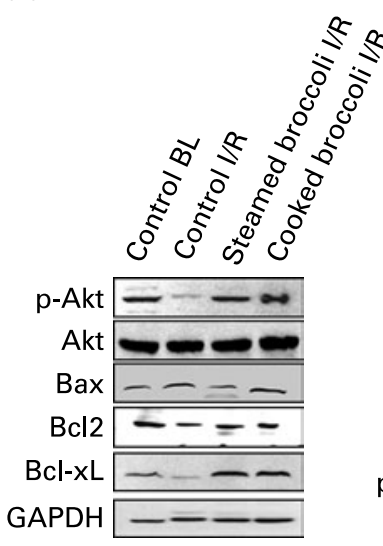

(b)

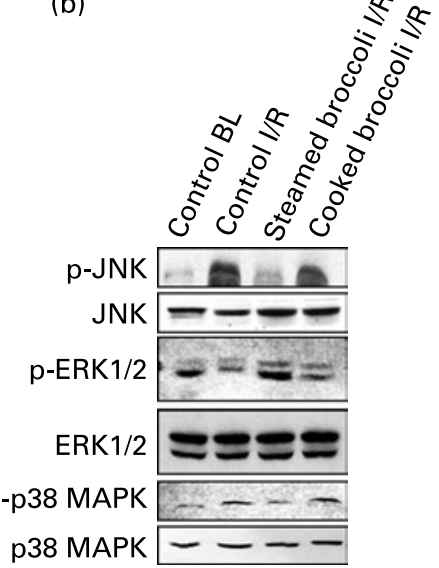

Fig. 6. Western blot analysis of p-Akt, Akt, Bax, Bcl2 and $\mathrm{Bcl}-\mathrm{xL}$ from the cytosolic fraction (a) and Western blot analysis of $\mathrm{p}$-c-Jun $\mathrm{N}$-terminal kinase (JNK), JNK, p-extracellular signal-regulated kinase (ERK1/2), ERK1/2, p-p38 mitogen-activated protein kinase (MAPK) and p38 MAPK from cytosolic fraction (b) of control baseline (baseline), ischaemic control, steamed and cooked broccoli-treated heart samples. Glyceraldehyde-6-phosphate dehydrogenase (GAPDH) was used as the loading control. Figures are representative images of three different groups, and each experiment was repeated at least thrice. I/R, ischaemia/reperfusion. two known mediators of redox signalling (Fig. 8(a)). As expected, ischaemia/reperfusion reduced the expression of both Nrf2 and HO-1 in case of non-treated control group. Broccoli treatment (both steamed and cooked) prevented the reduction of Nrf2 and HO-1 expression level due to ischaemia/reperfusion, but steamed broccoli could activate Nrf2 and HO-1 more effectively than that of the cooked broccoli.

\section{Effects of broccoli on superoxide dismutase activity}

Since broccoli is known to possess antioxidant properties ${ }^{(20,24)}$ and can induce the activity of $\operatorname{SOD}^{(25,26)}$, we examined the effects of broccoli before and after cooking on the activity

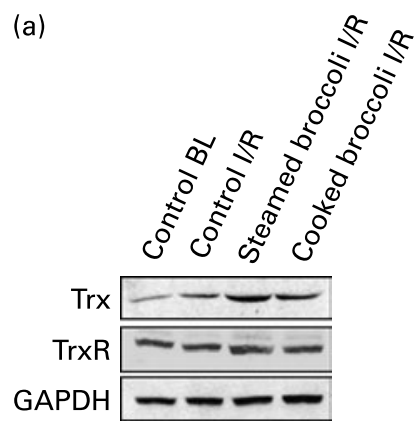

(b)

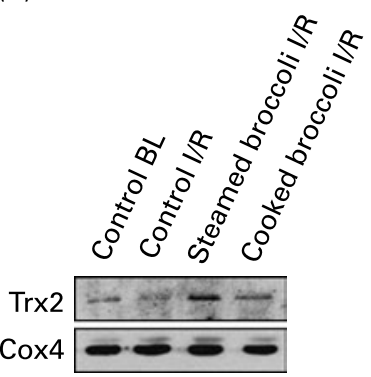

Fig. 7. Western blot analysis of Trx (whole), TrxR from the cytosolic fraction (a) and Trx2 from the mitochondrial fraction (b) of control baseline (BL), ischaemic control, steamed and cooked broccoli-treated heart samples. Glyceraldehyde-6-phosphate dehydrogenase (GAPDH) and Cox4 were used as loading control for cytosolic and mitochondrial fraction. Figures are representative images of three different groups, and each experiment was repeated at least thrice. $\mathrm{l} / \mathrm{R}$, ischaemia/reperfusion. 
(a)
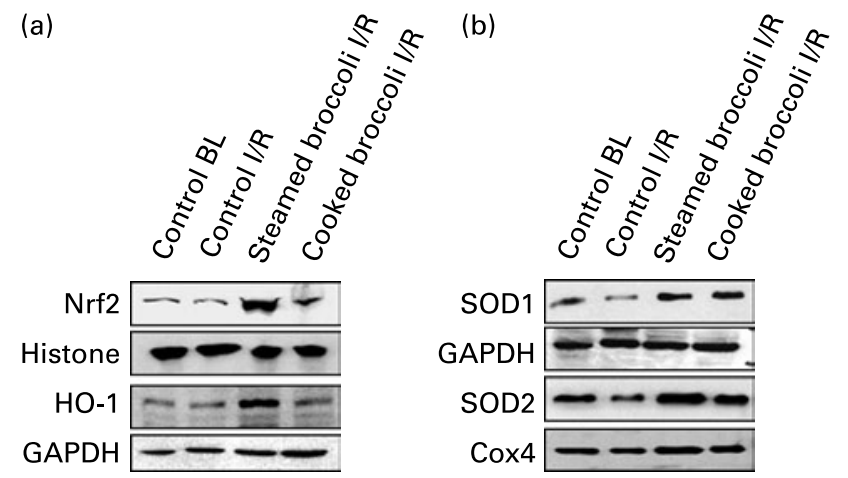

Fig. 8. Western blot analysis of NFE2 related factor 2 (Nrf2) from the nuclear fraction and haemoxygenase-1 $(\mathrm{HO}-1)$ from cytosolic fraction of control baseline $(\mathrm{BL})$, ischaemic control, steamed and cooked broccoli-treated heart samples. Histone and glyceraldehyde-6-phosphate dehydrogenase (GAPDH) were used as the loading control for nuclear and cytosolic fraction, respectively (a). Western blot analysis of Superoxide dismutase (SOD1) and SOD2 from cytosolic and mitochondrial fraction, respectively, of control, steamed and cooked broccoli-treated heart samples (b). GAPDH and Cox4 were used as loading control for cytosolic and mitochondrial fraction, respectively. Figures are representative images of three different groups, and each experiment was repeated at least thrice. I/R, ischaemia/reperfusion.

of SOD1 and SOD2. As shown in Fig. 8(b), ischaemia/reperfusion reduced the SOD1 and SOD2 levels in case of non-treated control group, but broccoli treatment prevented the reduction of SOD1 and SOD2 levels due to ischaemia/reperfusion injury. In case of SOD1 expression, both types of broccoli treatment prevented the loss of SOD1 protein due to ischaemia/reperfusion injury. But in case of SOD2 protein expression similar to other results, steamed broccoli showed superior effect over cooked broccoli.

\section{Discussion}

The results of the present study showed for the first time that steamed broccoli is a superior cardioprotective agent compared to well-cooked broccoli. The salient features of the study include: the feeding of steamed broccoli for a month (1) produces greater post-ischaemic ventricular recovery, lower myocardial infarction and reduced cardiomyocyte apoptosis compared to feeding cooked broccoli; (2) generates greater degree of survival signal by boosting anti-apoptotic $\mathrm{Bcl} 2$ and $\mathrm{Bcl}-\mathrm{xL}$ protein expression, increasing the phosphorylation of Akt and reducing the level of proapoptotic Bax protein; (3) induces higher degree of activation of survival protein ERK1/2 by phosphorylation and reduces death signal proteins JNK and p38 MAPK by decreasing their phosphorylation than that of the cooked counterpart; (4) induces Trx superfamily proteins like Trx, Trx 2 and TrxR more than that of the cooked broccoli; (5) generates greater degree of redox signalling by activating $\mathrm{Nrf} 2$ and the phase II enzyme HO-1; (6) induces higher level of SOD1 and SOD2 to make the first line of redox defense stronger.

Both steamed broccoli and cooked broccoli showed the protective effect against ischaemia/reperfusion-induced injury, but steamed broccoli revealed superior cardioprotective ability than that of cooked one. Thirty-minute ischaemia followed by 2 -h reperfusion reduced the ventricular function in case of non-treated control group. Broccoli prevented the reduction in ventricular function, but steamed broccoli improved the ventricular function more significantly than its cooked counterpart. For example, compared to control ischaemia/reperfusion, steamed broccoli increased the ventricular function including LVDP and aortic flow from 60 (SD 2.6) to 92.5 (SD 1.9) and from 13.66 (SD 1.4) to 33 (SD 1.29), respectively, while the cooked broccoli enhanced these functions from 60 (SD 2.6) to 83.16 (SD 0.87) and from 13.66 (SD 1.4) to 19.53 (SD 1.36), respectively. Consistent with the results of functional recovery, both steamed broccoli and cooked broccoli reduced the myocardial infarction significantly compared to non-treated control group. Again, steamed broccoli revealed smaller infarct size (18.82 (SD 1.24)\%) compared to cooked broccoli (23.44 (SD 1.00) \%), whereas in case of control group this value was 40.96 (SD 1.29) \%. The amount of cytochrome $\mathrm{c}$ was decreased in the non-treated ischaemic reperfused myocardium, which was completely reversed by both types of broccoli treatment indicating its ability to generate anti-apoptotic signal. Again, fresh broccoli showed better ability to generate anti-apoptotic signal. Consistent with these findings, the numbers of apoptotic cardiomyocytes were significantly reduced by both types of broccoli treatment. In case of control I/R group, the amount of apoptotic cardiomyocytes was 36.59 (SD 2.38) \%, and, in case of steamed and cooked broccolis, the amount of apoptotic cells was 17.74 (SD 1.13 ) \% and $22 \cdot 86$ (SD 2.28) \%, respectively.

The present results demonstrated that both types of broccoli preparations protected the heart from ischaemia/reperfusion injury either by upregulating survival signal or by downregulating death signal. For example, broccoli reduced pro-apoptotic Bax protein and induced the expression of anti-apoptotic $\mathrm{Bcl} 2$ and Bcl-xL proteins. Broccoli also activated the survival signal protein Akt via phosphorylation, and increased the activation of ERK1/2 and decreased the activation of p38 MAPK and JNK compared to control. P38 MAPK and JNK are known pro-apoptotic factors, while ERK1/2 is a well-known antiapoptotic factor. Thus, similar to the results of left ventricular functional parameter, infarct size and cardiomyocyte apoptosis, steamed broccoli consistently showed better abilities to activate anti-apoptotic Bcl2, Bcl-xL, Akt, ERK1/2 and/or to down-regulate pro-apoptotic Bax, p38 MAPK and JNK. It is known that broccoli can potentiate redox signalling by overexpressing Nrf2 and HO- ${ }^{(7)}$. Result of the present study also demonstrated that ischaemia/reperfusion injury disrupted the redox homeostasis by reducing Nrf2 and HO-1 levels. Both types of broccoli preparations prevented this reduction of Nrf2 and HO-1 due to ischaemia/reperfusion injury.

SOD acts as an antioxidant and exerts anti-inflammatory function in the body by dismutation of superoxide anions. There are two types of SOD: $\mathrm{Cu} / \mathrm{Zn}$ SOD (SOD1) and Mn SOD (SOD2). Each type of SOD plays a different role in protecting the body from oxidative damage. Ischaemia/reperfusion reduced the levels of SOD1 and SOD2 in rat myocardium, but broccoli protected the loss of SOD1 and SOD2, while steamed broccoli revealed superior ability to protect SOD system compared to cooked broccoli.

Although broccoli has been used as a vegetable for a long time, in modern society, it has become a vegetable of choice because of its high nutritious value and diverse health benefits. Along with other cruciferous vegetables, broccoli possesses anticancer properties. Studies have shown that broccoli consumption reduces the risk of several types of cancer including 
lung, stomach and breast cancers ${ }^{(27-29)}$. Broccoli juices (from leaves) can also prevent skin diseases ${ }^{(2)}$. It has also potential antioxidant properties ${ }^{(20,24)}$. A recent clinical study has suggested that consumption of fresh broccoli sprouts $(100 \mathrm{~g} / \mathrm{d})$ for a week reduced LDL and total cholesterol and increased HDL cholesterol ${ }^{(4)}$. Another related study showed that broccoli was strongly associated with reduced risk of $\mathrm{CHD}^{(30)}$. A recent study has demonstrated that broccoli consumption could prevent the reduction of mRNA levels and protein level of Trx super family members due to ischaemic reperfusion injury ${ }^{(7)}$. Sulphoraphane present in broccoli is a well-known inducer of Trx reductase ${ }^{(23)}$, which helps Trx to maintain its reduced state which in turn maintains the redox homeostasis of the mammalian system. The present study demonstrated that ischaemia/reperfusion injury reduced the level of Trx superfamily proteins, whereas broccoli prevented the reduction of Trx proteins, steamed broccoli being superior over its cooked counter part. In the present study, the authors demonstrated that broccoli treatment could improve cardiac function, reduce myocardial infraction and cardiomyocyte apoptosis after ischaemic reperfusion injury. Recently, a group of researchers from the United Kingdom reported that eating broccoli could reverse diabetes-induced damage to blood vessels in the heart ${ }^{(31)}$.

The health benefits of broccoli consumption are most likely mediated through the 'bioactive compounds' present in broccoli, which induce a variety of physiological responses including acting as antioxidants, regulating enzymes and controlling apoptosis and the cell cycle proteins. Epidemiological and experimental studies have shown that broccoli contains good amount of bioactive components including flavonoids, minerals, vitamins and also is a good source of glucosinolate ${ }^{(32,33)}$. Although all these bioactive compounds can play an important role in providing health benefits, sulphoraphane is likely to play a crucial role. Sulphoraphane has been found to have protective effects against carcinogen-induced tumourogenesis $^{(11,34)}$. It is a natural inducer of phase II enzymes in both human and animals ${ }^{(13,14)}$. In in vitro assays, sulphoraphane was found to induce quinone reductase, glutathione $S$-reductase and UDP-glucuronosyltransferase ${ }^{(35)}$. In these studies, sulphoraphane could increase both UGT1A1 and UGTA1 mRNA levels in hepG2 and HT29 cells ${ }^{(35)}$. Although sulphoraphane is not a direct-acting antioxidant or pro-oxidant, there is substantial and growing evidence that sulphoraphane administration acts indirectly to increase the antioxidant capacity of animal cells ${ }^{(36)}$. Sulphoraphane can attenuate oxidative stress, hypertension and inflammation in the cardiovascular system $^{(37)}$. A recent study has also suggested that broccoli could protect mammalian heart from ischaemia/reperfusion injury via the activation of Trx superfamily due to the presence of sulphoraphane ${ }^{(7)}$. Also, sulphoraphane can activate the transcription factor $\mathrm{Nrf} 2$ and induces phase 2 and antioxidant enzyme in human keratinocytes in culture ${ }^{(38,39)}$. Interestingly enough, broccoli does not contain sulphoraphane, but contains glucosinolate, becomes hydrolysed into sulphoraphane due to the enzymatic activity of myrosinase. Cooking reduces glucosinolate levels by approximately 30-60\%, depending on the cooking method, cooking intensity and on the type of cooking ${ }^{(40)}$. On the other hand, studies showed that both myrosinase enzyme and sulphoraphane degraded at high temperature ${ }^{(18,19)}$. One study by Lopez-Berenguer demonstrated that bioactive compounds in broccoli, such as vitamin $\mathrm{C}$, phenolic compounds and glucosinolate concentration reduced after microwave cooking ${ }^{41)}$. Except for glucosinolate and sulphoraphane, most of the bioactive compounds including antioxidants and minerals are preserved in cooked broccoli ${ }^{(41)}$. A study also showed that cooking has no effect on the antioxidant properties of broccoli ${ }^{(20)}$. Thus, it is quite natural that broccoli retains many of its cardioprotective functions even after cooking.

In summary, the results of the present study clearly demonstrated for the first time that steamed broccoli is a better cardioprotectant than cooked broccoli as evidenced by better cardiac function, reduced myocardial infarction and decreased cardiomyocyte apoptosis. In concert, steamed broccoli documented significantly, improved survival signal and reduced death signal compared to cooked broccoli. As discussed above, it appears that cooked broccoli loses sulphoraphane and thus loses its ability to generate Trx, which presumably provides additional cardioprotection in steamed broccoli.

\section{Acknowledgements}

The present study was supported by National Institutes of Health (NIH) grants HL34360, HL22559 and HL33889 to D. K. D., S. M., I. L. and D. R. did the experiments, S. M. and D. K. D. wrote the manuscript; U. R., H. G. and D. K. D. designed the study.

We have no conflicts of interest.

\section{References}

1. Gill CI, Haldar S, Porter S, et al. (2004) The effect of cruciferous and leguminous sprouts on genotoxicity, in vitro and in vivo. Cancer Epidemiol Biomarkers Prev 13, 1199-1205.

2. Guarrera PM (2005) Traditional phytotherapy in Central Italy (Marche, Abruzzo, and Latium). Fitoterapia 76, 1-25.

3. Eberhardt MV, Kobira K, Keck AS, et al. (2005) Correlation analyses of phytochemical composition, chemical, and cellular measures of antioxidant activity of broccoli (Brassica oleracea L. Var. italica). J Agric Food Chem 53, 7421-7431.

4. Murashima M, Watanabe S, Zhuo XG, et al. (2004) Phase 1 study of multiple biomarkers for metabolism and oxidative stress after one-week intake of broccoli sprouts. Biofactors 22, 271-275.

5. Perocco P, Bronzetti G, Canistro D, et al. (2006) Glucoraphanin, the bioprecursor of the widely extolled chemopreventive agent sulforaphane found in broccoli, induces phase-I xenobiotic metabolizing enzymes and increases free radical generation in rat liver. Mutat Res 595, 125-136.

6. Sesso HD, Gaziano JM, Liu S, et al. (2003) Flavonoid intake and the risk of cardiovascular disease in women. Am J Clin Nutr 77, 1400-1408.

7. Mukherjee S, Gangopadhyay H \& Das DK (2008) Broccoli: a unique vegetable that protects mammalian hearts through the redox cycling of the thioredoxin superfamily. J Agric Food Chem 56, 609-617.

8. Matusheski NV, Swarup R, Juvik JA, et al. (2006) Epithiospecifier protein from broccoli (Brassica oleracea L. ssp. italica) inhibits formation of the anticancer agent sulforaphane. J Agric Food Chem 54, 2069-2076.

9. Finley JW (2003) Reduction of cancer risk by consumption of selenium-enriched plants: enrichment of broccoli with selenium increases the anticarcinogenic properties of broccoli. J Med Food 6, 19-26. 
10. Finley JW, Sigrid-Keck A, Robbins RJ, et al. (2005) Selenium enrichment of broccoli: interactions between selenium and secondary plant compounds. J Nutr 135, 1236-1238.

11. Zhang Y, Kensler TW, Cho CG, et al. (1994) Anticarcinogenic activities of sulforaphane and structurally related synthetic norbornyl isothiocyanates. Proc Natl Acad Sci U S 91, 3147-3150.

12. Fahey JW, Haristoy X, Dolan PM, et al. (2002) Sulforaphane inhibits extracellular, intracellular, and antibiotic-resistant strains of Helicobacter pylori and prevents benzo[a]pyreneinduced stomach tumors. Proc Natl Acad Sci U A 99, 7610-7615.

13. Talalay P (2000) Chemoprotection against cancer by induction of phase 2 enzymes. Biofactors 12, 5-11.

14. Prochaska HJ, Santamaria AB \& Talalay P (1992) Rapid detection of inducers of enzymes that protect against carcinogens. Proc Natl Acad Sci U S A 89, 2394-2398.

15. Tanito M, Masutani H, Kim YC, et al. (2005) Sulforaphane induces thioredoxin through the antioxidant-responsive element and attenuates retinal light damage in mice. Invest Ophthalmol Vis Sci 46, 979-987.

16. Fenwick GR, Heaney RK \& Mullin WJ (1983) Glucosinolates and their breakdown products in food and food plants. Crit Rev Food Sci Nutr 18, 123-201.

17. Rushmore TH, Morton MR \& Pickett CB (1991) The antioxidant responsive element. Activation by oxidative stress and identification of the DNA consensus sequence required for functional activity. J Biol Chem 266, 11632-11639.

18. Ludikhuyze L, Ooms V, Weemaes C, et al. (1999) Kinetic study of the irreversible thermal and pressure inactivation of myrosinase from broccoli (Brassica oleracea L. Cv. italica). J Agric Food Chem 47, 1794-1800.

19. Van Eylen D, Oey I, Hendrickx M, et al. (2007) Kinetics of the stability of broccoli (Brassica oleracea $\mathrm{Cv}$. Italica) myrosinase and isothiocyanates in broccoli juice during pressure/temperature treatments. J Agric Food Chem 55, 2163-2170.

20. Lin CH \& Chang CY (2005) Textural change and antioxidant properties of broccoli under different cooking treatments. Food Chem 90, 9-15.

21. Wu L, Noyan Ashraf MH, Facci M, et al. (2004) Dietary approach to attenuate oxidative stress, hypertension, and inflammation in the cardiovascular system. Proc Natl Acad Sci U S A 101, 7094-7099.

22. Mukherjee S, Lekli I, Das M, et al. (2008) Cardioprotection with alpha-tocopheryl phosphate: amelioration of myocardial ischemia reperfusion injury is linked with its ability to generate a survival signal through Akt activation. Biochim Biophys Acta 1782, 498-503.

23. Das DK (2004) Thioredoxin regulation of ischemic preconditioning. Antioxid Redox Signal 6, 405-412.

24. Hintze KJ, Wald K \& Finley JW (2005) Phytochemicals in broccoli transcriptionally induce thioredoxin reductase. J Agric Food Chem 53, 5535-5540.

25. Piao XL, Kim HY, Yokozawa T, et al. (2005) Protective effects of broccoli (Brassica oleracea) and its active components against radical-induced oxidative damage. J Nutr Sci Vitaminol (Tokyo) 51, 142-147.
26. Li YM, Chan HY, Huang Y, et al. (2007) Broccoli (Brassica oleracea var. botrytis L.) improves the survival and up-regulates endogenous antioxidant enzymes in Drosophila melanogaster challenged with reactive oxygen species. J Sci Food Agric 88, 499-506.

27. Villa-Cruz V, Davila J, Viana MT, et al. (2009) Effect of broccoli (Brassica oleracea) and its phytochemical sulforaphane in balanced diets on the detoxification enzymes levels of tilapia (Oreochromis niloticus) exposed to a carcinogenic and mutagenic pollutant. Chemosphere 74, 1145-1151.

28. Brandi G, Schiavano GF, Zaffaroni N, et al. (2005) Mechanisms of action and antiproliferative properties of Brassica oleracea juice in human breast cancer cell lines. J Nutr 135, 1503-1509.

29. Jackson SJ \& Singletary KW (2004) Sulforaphane inhibits human MCF-7 mammary cancer cell mitotic progression and tubulin polymerization. J Nutr 134, 2229-2236.

30. Cohen JH, Kristal AR \& Stanford JL (2000) Fruit and vegetable intakes and prostate cancer risk. J Natl Cancer Inst 92, 61-68.

31. Yochum L, Kushi LH, Meyer K, et al. (1999) Dietary flavonoid intake and risk of cardiovascular disease in postmenopausal women. Am J Epidemiol 149, 943-949.

32. Xue M, Qian Q, Adaikalakoteswari A, et al. (2008) Activation of NF-E2-related factor-2 reverses biochemical dysfunction of endothelial cells induced by hyperglycemia linked to vascular disease. Diabetes 57, 2809-2817.

33. Jeffery EH, Brown AF, Kurilich AC, et al. (2003) Variation in content of bioactive components in broccoli. J Food Compos Anal 16, 323-330.

34. Finley JW, Ip C, Lisk DJ, et al. (2001) Cancer-protective properties of high-selenium broccoli. J Agric Food Chem 49, 2679-2683.

35. Fahey JW, Zhang Y \& Talalay P (1997) Broccoli sprouts: an exceptionally rich source of inducers of enzymes that protect against chemical carcinogens. Proc Natl Acad Sci U S A 94, 10367-10372.

36. Basten GP, Bao Y \& Williamson G (2002) Sulforaphane and its glutathione conjugate but not sulforaphane nitrile induce UDP-glucuronosyl transferase (UGT1A1) and glutathione transferase (GSTA1) in cultured cells. Carcinogenesis 23, 1399-1404.

37. Fahey JW \& Talalay P (1999) Antioxidant functions of sulforaphane: a potent inducer of phase II detoxication enzymes. Food Chem Toxicol 37, 973-979.

38. Wagner AE, Ernst I, Iori R, et al. (2009) Sulforaphane but not ascorbigen, indole-3-carbinole and ascorbic acid activates the transcription factor Nrf2 and induces phase-2 and antioxidant enzymes in human keratinocytes in culture. Exp Dermatol, (in press).

39. Lee JS \& Surh YJ (2005) Nrf2 as a novel molecular target for chemoprevention. Cancer Lett 28, 171-184.

40. Rungapamestry V, Duncan AJ, Fuller Z, et al. (2007) Effect of cooking brassica vegetables on the subsequent hydrolysis and metabolic fate of glucosinolates. Proc Nutr Soc 66, 69-81.

41. Lopez-Berenguer C, Carvajal M, Moreno DA, et al. (2007) Effects of microwave cooking conditions on bioactive compounds present in broccoli inflorescences. J Agric Food Chem 55, 10001-10007. 